\title{
Quadriceps Muscle Strain among Athletes: Case report
}

Ahmad Atiq Alharbi*, Dareen Ibrahim Rednah

Department Orthopedic Surgery, My Clinic Mohammadiya Hospital, Jeddah, Saudi Arabia

*Corresponding author: Ahmad Atiq Alharbi, Mobile: +966555071126, E-Mail: Alrehaily.ahmad@gmail.com

\begin{abstract}
Background: In this case report we present a case of both vastus lateralis and intermedialis sprain in basketball athlete young male who presented to the clinic with loss of knee motion, anterior knee pain and tense anterior thigh.

Case report: We present a young male in his early twenties, who was engaged in recreational sports activities regularly and practice basketball as professional player. He sustained a direct blow to his right thigh by direct trauma while playing basketball, and then suddenly experienced severe pain with minor tingling sensation. No other injuries were noted at the time. Later, he developed anterior knee pain and significant loss of knee motion and by the end of the day his anterior thigh became tense with very severe pain.

Conclusion: The quadriceps injury among athletes is common as combined injury of the two heads of quadriceps and very rare as an isolated one head of quadriceps and the best treatment of such injury is to prevent it.
\end{abstract}

Keywords: Quadriceps muscle strain, Lateralis muscle, Athletes.

\section{INTRODUCTION}

The vastus lateralis muscle is located on the lateral side of the thigh. This muscle is the largest of the quadriceps which includes: rectus femoris, vastus intermedius, and vastus medialis. Together, the quadriceps acts on the knee and hip to promote movement as well as strength and stability. They provide power for and absorb the impact of daily activities such as walking, running, and jumping ${ }^{(1)}$.

\section{CASE REPORT}

We present a young male in his early twenties, who engages in recreational sports activities regularly and practice basketball as professional player attending at My Clinic, Mohammadiya Hospital. He sustained a direct blow to his right thigh by direct trauma while playing basketball, and then suddenly experienced severe pain with minor tingling sensation. No other injuries were noted at the time. Later, he developed anterior knee pain and significant loss of knee motion. By the end of the day his anterior thigh became tense with very severe pain.

Upon examining the patient in the office, he was mildly distressed because of the pain, his vitals were unremarkable. Locally there was a moderate swelling over the anterior aspect of the thigh with some redness, tender on palpitation with a noticeable depression over distal portion of his thigh, which gave us false impression of quadriceps tendon partial rupture found lateral to be 2 nd to hematoma collection proximally.
The range of motion of the knee was around $0-80$ degrees. Knee motion with hip extended was significantly compromised but with flexion of the hip motion of the knee was improved mildly. Hip and ankle motion were normal, distal pulses were palpable with good capillary refill and no neurological deficits were noted.

His injured leg was mild colder than the other leg, which increased the suspicion of hematoma anterior thigh that compromised one of the blood supplies to lower extremity. Urgent Arterial and venous Doppler study were done that showed good flow with no significant stenosis, however thick heterogeneous anterior thigh muscles were seen.

Plain radiographs showed degenerative changes of both hips joints, no other osseous or soft tissue findings were noted; an MRI was the next step. The MRI report stated that the Vastus Lateralis and Intermedialis muscles were severely strained with evident intramuscular hematoma.

Our initial step in managing the patient condition included close monitoring of the patient for compartment syndrome with elevation, ice and pain medication improved the swelling and the motion. We started for aggressive quad muscle strengthening and hamstring strengthening protocol. After 14 session of physiotherapy, the motion of the patient returned to normal base and the strength of the muscle was much improved. 

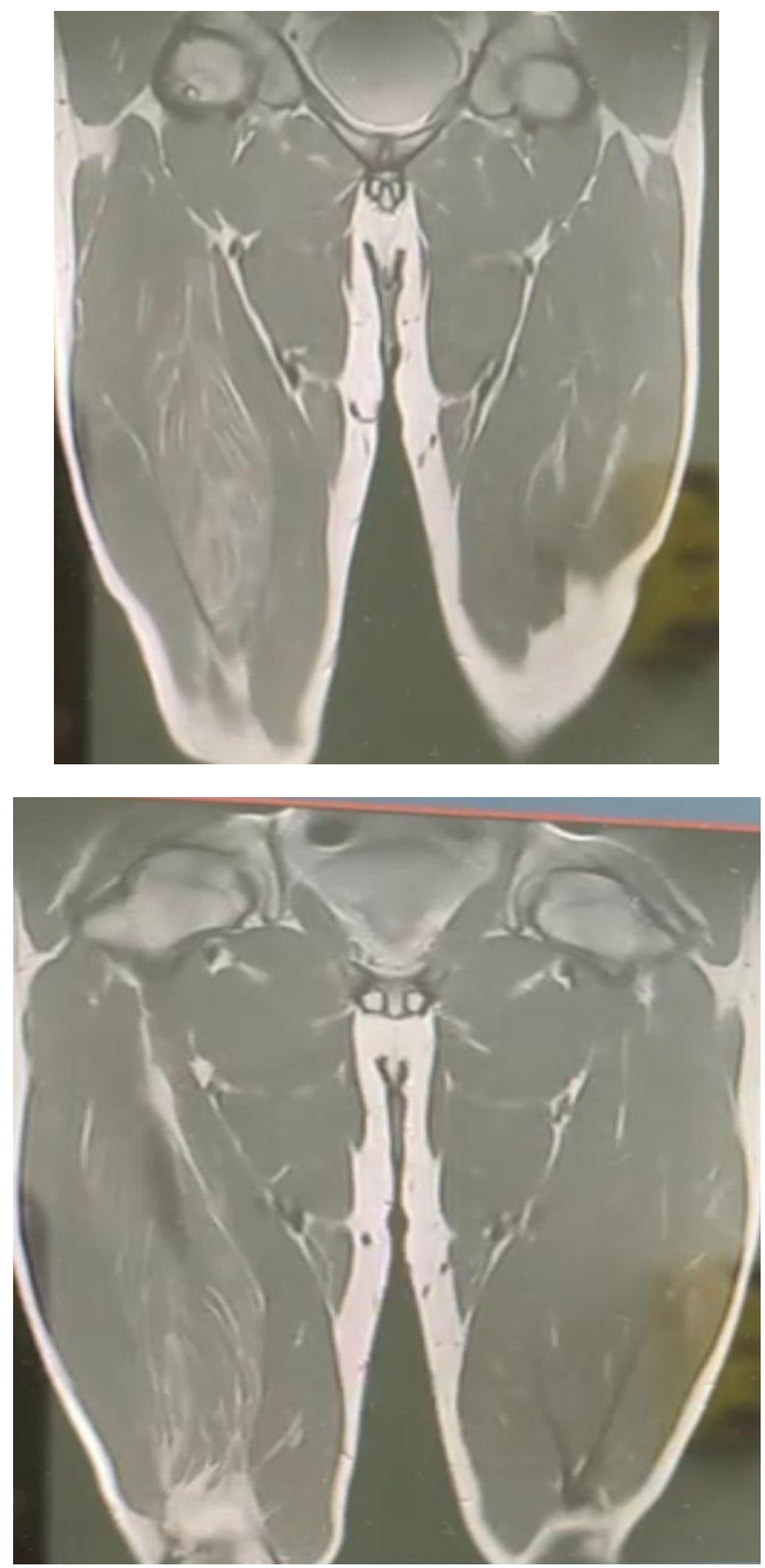

Figure (1): MRI sequences showed that the Vastus Lateralis and Intermedius muscles were severely strained with evidence of intramuscular collection.

Declaration of patient consent:

An approval of the study was obtained from Mohammadiya Hospital, Jeddah, Saudi Arabia Academic and Ethical Committee. The patient was informed that his case was took as case-report for publishing and he accepted. This work has been carried out in accordance with The Code of Ethics of the World Medical Association (Declaration of Helsinki) for studies involving humans. 


\section{DISCUSSION}

Quadriceps muscle contain four muscles: vastus lateralis which originates from the lateral part of intertrochanteric line, margin of greater trochanter, lateral margin of gluteal tuberosity, lateral lip of the linea asparta and inserted into quadriceps tendon and vastus medialis that originates from the medial part of intertrochanteric line, pectineal line, medial lip of the linea aspera, medial supracondylar line and inserted into medial border of the patella through the quadriceps tendon. In addition, vastus intermedialis that originates from proximal two third of anterolateral femur and inserted into lateral side of the patella through quadriceps tendon and finally the rectus femoris muscle including straight head, which originates from the anterior inferior iliac spine and reflected head that originates from the ilium just superior to the acetabulum and inserted into the quadriceps tendon. All four heads act as hip flexors and knee extensors ${ }^{(1)}$.

The incidence of quadriceps injury was reported by Eckard et al. (2) among the national collegiate athletes association was found to be less common than hamstring injury three times more common in competitive female athletes than in males. In preseason training in non-contact injuries $63 \%$ and $22 \%$ in overuse and recurrence rate about $7.2 \%$ in basketball among female and football among male ${ }^{(2)}$.

In this case discussion, we presented a case vastus lateralis and intermedialis injury in basketball competitive athletes. Isolated vastus lateralis injury was reported in literature as two different case reports one by Frank et al. ${ }^{(3)}$ as it is more common in male athletes and when it is avulsed, it is recommended to undergo surgical fixation to preserve patella tracking.

In literature there were no significant reported cases about vastus intermedialis sprain. Souza et al. ${ }^{(4)}$, Voight et al. ${ }^{(5)}$ and Cowan et al. ${ }^{(6)}$ suggested, based on electromyographic studies, that altered activation patterns can disturb patellofemoral joint dynamics. LaBore and Weiss ${ }^{(7)}$ suggested in their review that vastus lateralis sprain affected the patello-femoral tracking and patient that is presented with patellofemoral anterior pain can be treated with VMO exercise to overcome sprained vastus lateralis.

Treatment of quadriceps strain includes ice and strengthening of the muscles but the most important is to prevent such injuries especially in athletes to avoid delay in recovery and to return to sport. Many protocols were published, one of these protocols was published by Daneshjoo et al. ${ }^{(8)}$ as they recommended both FIFA +11 program and the HarmoKnee training prevention programs which combine strength, neuromuscular control, balance and proper motion patterns without using special equipment. It can improve isometric quadriceps strength.

\section{CONCLUSION}

The quadriceps injury among athletes is common as combined injury of two heads of quadriceps and very rare as an isolated one head of quadriceps and best treatment of such injury is to prevent it.

Financial support and sponsorship: Nil. Conflict of interest: Nil.

\section{REFERENCES}

1. Gray H, Drake R, Vogl W et al. (2010): Gray's anatomy for Students 2nd ed. Philadelphia: Churchill Livingstone/Elsevier, $\quad$ Pp: 1180. https://www.elsevier.com/ books/grays-anatomy-forstudents/drake/978-0-323-39304-1

2. Eckard T, Kerr Z, Padua D et al. (2017): Epidemiology of Quadriceps Strains in National Collegiate Athletic Association Athletes, 2009-2010 through 2014-2015. J Athl Train., 52 (5): 474-481.

3. Frank J, Riedel M, McCormick F et al. (2013): Isolated vastus lateralis tendon avulsion. Am J Orthop (Belle Mead NJ), 42 (10): 464-5.

4. Souza D, Gross M (1991): Comparison of vastus medialis obliquus: vastus lateralis muscle integrated electromyographic ratios be- tween healthy subjects and patients with patellofemoral pain. Phys Ther., 71: 310-20.

5. Voight M, Weider D (1991): Comparative reflex response time of vastus medialis obliquus and vastus lateralis in normal subjects and subjects with extensor mechanism dysfunction. Am J Sports Med., 19: 131-7.

6. Cowan S, Bennell K, Hodges $P$ et al. (2001): Delayed onset of electromyographic activity of vastus medialis obliquus relative to vastus lateralis in subjects with patellofemoral pain syndrome. Arch Phys Med Rehabil., 82: 183-9.

7. LaBore A, Weiss D (2003): Vastus lateralis strain associated with patellofemoral pain syndrome: a report of 2 cases. Arch Phys Med Rehabil., 84 (4): 613-5.

8. Daneshjoo A, Rahnama N, Mokhtar A et al. (2013): Effectiveness of injury prevention programs on developing quadriceps and hamstrings strength of young male professional soccer players. J Hum Kinet., 39: 115-125. 